Supporting Information

\title{
Atomic Polarizabilities for Interactive Dipole Induction Models
}

Jacob M. Litman, ${ }^{\dagger}$ Chengwen Liu, ${ }^{\dagger}$ and Pengyu Ren*

Department of Biomedical Engineering, The University of Texas at Austin, Austin, TX 78712, USA

$\dagger$ Authors that contributed equally to this work

*Corresponding author. Email: pren@mail.utexas.edu 

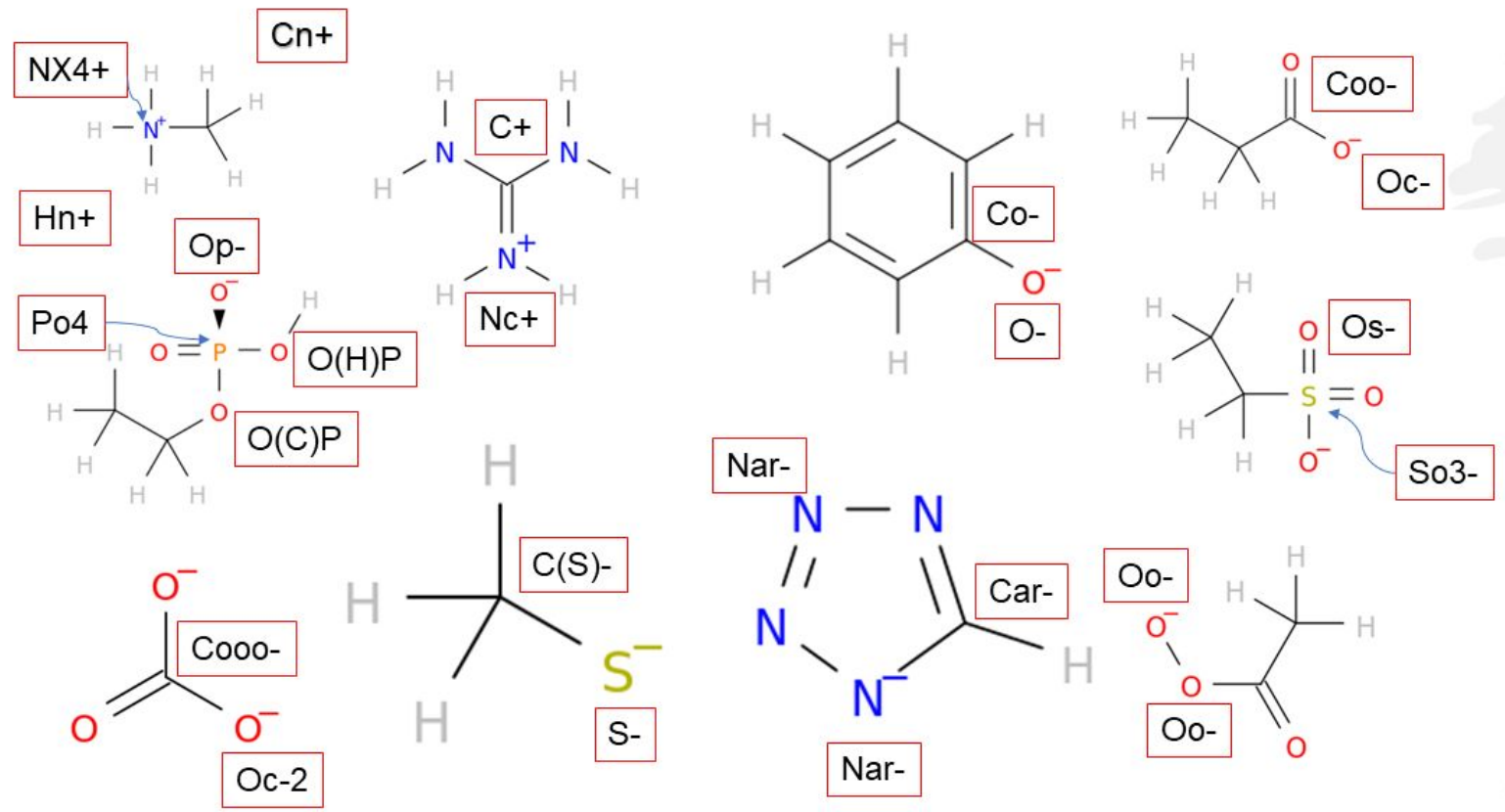

Figure S1. An illustration of the atom types for charged molecules. The same atom type is assigned to chemically equivalent atoms in a molecule (for example three nitrogen atoms are all "Nc+" in guanidinium ion). For the remaining atoms in a molecule, SMARTS pattern matching for the neutral molecules is applied. 
Table S1. Atomic polarizabilities of the current AMOEBA model.

\begin{tabular}{llll}
\hline Index & SMARTS & $\boldsymbol{\alpha}\left(\AA^{3}\right)$ & Description \\
\hline 1 & {$[\# 1]$} & 0.496 & Hydrogen \\
2 & {$[\# 1][\mathrm{c}, \mathrm{n}]$} & 0.696 & Hydrogens on aromatic carbon/nitrogen \\
3 & {$[\mathrm{C}]$} & 1.334 & Non-aromatic carbon \\
4 & {$[\mathrm{c}]$} & 1.750 & Aromatic carbon \\
5 & {$[\# 7]$} & 1.073 & Nitrogen \\
6 & {$[\# 8]$} & 0.837 & Oxygen \\
7 & {$[\# 16]$} & 2.800 & Sulfur \\
8 & {$[\# 15]$} & 1.788 & Phosphorus \\
9 & {$[\# 9]$} & 0.600 & Florine \\
10 & {$[\# 17]$} & 2.500 & Chloride \\
11 & {$[\# 35]$} & 3.595 & Bromine \\
12 & {$[\# 53]$} & 5.705 & Iodine \\
\hline
\end{tabular}


Table S2. Atom types are defined by SMARTS strings for the neutral molecules. The first atom in the smarts string is of interest. The more detailed matching takes the priority and overrides a courser matching for a specific atom.

\begin{tabular}{|c|c|c|c|}
\hline Element & SMARTS & Atom type & Description \\
\hline \multirow[t]{12}{*}{$\mathrm{H}$} & {$[\mathrm{H}]$} & $\mathrm{H}^{*}$ & wild matching $\mathrm{H}$ \\
\hline & {$[\mathrm{H}][\mathrm{C}]$} & Hnonpol & $\mathrm{H}$ on non-aromatic $\mathrm{C}$ \\
\hline & {$[\mathrm{H}][\mathrm{O}]$} & Hpolar & $\mathrm{H}$ on non-aromatic $\mathrm{O}$ \\
\hline & {$[\mathrm{H}][\mathrm{N}]$} & Hpolar & $\mathrm{H}$ on non-aromatic $\mathrm{N}$ \\
\hline & {$[\mathrm{H}][\mathrm{S}]$} & Hpolar & $\mathrm{H}$ on non-aromatic $\mathrm{S}$ \\
\hline & {$[\mathrm{H}][\mathrm{NH} 3]$} & Hpolar & $\mathrm{H}$ on ammonia \\
\hline & {$[\mathrm{H}][\mathrm{F}, \mathrm{Cl}, \mathrm{Br}, \mathrm{I}]$} & Hpolar & $\mathrm{H}$ on $\mathrm{HX}(\mathrm{X}=\mathrm{F}, \mathrm{Cl}, \mathrm{Br}$, and $\mathrm{I})$ \\
\hline & {$[\mathrm{H}][\mathrm{c}]$} & Har & $\mathrm{H}$ on aromatic $\mathrm{C}$ \\
\hline & {$[\mathrm{H}][\mathrm{n}]$} & Har & $\mathrm{H}$ on aromatic $\mathrm{N}$ \\
\hline & {$[\mathrm{H}][\mathrm{o}]$} & Har & $\mathrm{H}$ on aromatic $\mathrm{O}$ \\
\hline & {$[\mathrm{H}][\mathrm{C}]=[\mathrm{C}]$} & Har & $\mathrm{H}$ on $\mathrm{C}=\mathrm{C}$ system \\
\hline & {$[\mathrm{H}][\mathrm{OH} 2]$} & HW & $\mathrm{H}$ on water \\
\hline \multirow[t]{16}{*}{$\mathrm{C}$} & {$[\mathrm{C}]$} & $\mathrm{C}^{*}$ & wild matching $\mathrm{C}$ \\
\hline & {$[\$([C][C])]$} & Cnonpol & $\mathrm{C}$ on non-aromatic $\mathrm{C}$ \\
\hline & {$[\mathrm{C}][\mathrm{c}]$} & Cnonpol & $\mathrm{C}$ connected to aromatic $\mathrm{C}$ \\
\hline & {$[\mathrm{C}][\mathrm{O}]$} & Cpolar & $\mathrm{C}$ on non-aromatic $\mathrm{O}$ \\
\hline & {$[\mathrm{C}][\mathrm{o}]$} & Cpolar & $\mathrm{C}$ on aromatic $\mathrm{O}$ \\
\hline & {$[\mathrm{C}][\mathrm{N}]$} & Cpolar & $\mathrm{C}$ on non-aromatic $\mathrm{N}$ \\
\hline & {$[\mathrm{C}][\mathrm{n}]$} & Cpolar & $\mathrm{C}$ on aromatic $\mathrm{N}$ \\
\hline & {$[\mathrm{C}][\mathrm{F}, \mathrm{Cl}, \mathrm{Br}, \mathrm{I}]$} & Cpolar & $\mathrm{C}$ connected to $\mathrm{F}, \mathrm{Cl}, \mathrm{Br}$, and $\mathrm{I}$ \\
\hline & {$[\mathrm{C}]=[\mathrm{N}]$} & Cpolar & $\mathrm{C}$ connected to $\mathrm{N}$ via double bond \\
\hline & {$[\mathrm{C}][\mathrm{S}]$} & Cpolar & $\mathrm{C}$ on $\mathrm{S}$ \\
\hline & {$[\mathrm{c}]$} & Car & C on aromatic system \\
\hline & {$[\mathrm{CX} 3](=\mathrm{O})$} & Car & $\mathrm{C}$ on carbonyl $\mathrm{O}$ \\
\hline & {$[\$([\mathrm{CX} 3]=[\mathrm{CX} 3])]$} & Car & $\mathrm{C}$ on $\mathrm{C}=\mathrm{C}$ \\
\hline & {$[\mathrm{C}]([\mathrm{C}]=[*])([\mathrm{C}]=[*]$} & Car & $\mathrm{C}$ on conjugated systems \\
\hline & {$[\mathrm{CH} 4]$} & Cch4 & C on methane \\
\hline & {$[\$([C] \#[*])]$} & Csp & $\mathrm{C}$ on triple bond, e.g. $\mathrm{C} \# \mathrm{C}, \mathrm{C} \# \mathrm{~N}$ \\
\hline \multirow[t]{11}{*}{$\mathrm{N}$} & {$[\mathrm{N}]$} & $\mathrm{N}^{*}$ & wild matching Nitrogen \\
\hline & {$[\mathrm{N}][\mathrm{C}]$} & Nnonpol & $\mathrm{N}$ on non-aromatic $\mathrm{C}$ \\
\hline & {$[\mathrm{N}][\mathrm{c}]$} & Nnonpol & $\mathrm{N}$ connected to aromatic $\mathrm{C}$ via single bond \\
\hline & {$[\mathrm{N}]=[\mathrm{c}]$} & Nnonpol & $\mathrm{N}$ connected to aromatic $\mathrm{C}$ via double bond \\
\hline & {$[\mathrm{N}][\mathrm{S}]$} & Npolar & $\mathrm{N}$ connected to $\mathrm{S}$ \\
\hline & {$[\$([N][N])]$} & Npolar & $\mathrm{N}$ connected to non-aromatic $\mathrm{N}$ via single bond \\
\hline & {$[\mathrm{N}]=[\mathrm{N}]$} & Npolar & $\mathrm{N}$ connected to non-aromatic $\mathrm{N}$ via double bond \\
\hline & {$[\mathrm{N}][\mathrm{n}]$} & Npolar & $\mathrm{N}$ connected to aromatic $\mathrm{N}$ via single bond \\
\hline & {$[\mathrm{n}]$} & Nar & $\mathrm{N}$ on aromatic system \\
\hline & {$[\mathrm{N}]=[\mathrm{C}]$} & Nar & $\mathrm{N}$ on double bond $\mathrm{N}=\mathrm{C}$ \\
\hline & {$[\mathrm{NX} 3]([\mathrm{CX} 3](=\mathrm{O}))$} & Nsp2 & $\mathrm{N}$ on amide \\
\hline
\end{tabular}




\begin{tabular}{|c|c|c|c|}
\hline & $\begin{array}{l}{[\mathrm{N}] \#[*]} \\
{[\mathrm{NX} 3 \mathrm{H} 3]}\end{array}$ & $\begin{array}{l}\text { Nsp } \\
\text { Nnh3 }\end{array}$ & $\begin{array}{l}\mathrm{N} \text { of triple bond, } \mathrm{N} \# \mathrm{~N}, \mathrm{~N} \# \mathrm{C} \text {, etc } \\
\mathrm{N} \text { on ammonia }\end{array}$ \\
\hline \multirow[t]{13}{*}{$\mathrm{O}$} & {$[\mathrm{O}]$} & $\mathrm{O}^{*}$ & wild matching $\mathrm{O}$ \\
\hline & {$[\mathrm{O}][\mathrm{CX} 4]$} & Ononpol & O on non-polar $\mathrm{C}$ \\
\hline & {$[\mathrm{O}][\mathrm{c}]$} & Ononpol & $\mathrm{O}$ connected to aromatic $\mathrm{C}$ via single bond \\
\hline & {$[\mathrm{O}]=[\mathrm{c}]$} & Ononpol & $\mathrm{O}$ connected to aromatic $\mathrm{C}$ via double bond \\
\hline & {$[\mathrm{O}][\mathrm{N}]$} & Opolar & $\mathrm{O}$ connected to non-aromatic $\mathrm{N}$ \\
\hline & {$[\mathrm{O}][\mathrm{n}]$} & Opolar & $\mathrm{O}$ connected to aromatic $\mathrm{N}$ \\
\hline & {$[0]$} & Oar & $\mathrm{O}$ on aromatic system \\
\hline & {$[\mathrm{OX} 2][\mathrm{CX} 3]$} & Oar & O connected to carbonyl group \\
\hline & {$[\mathrm{OX} 1]=[\mathrm{CX} 3]$} & Osp2c & O on carbonyl group \\
\hline & {$[\mathrm{OX} 2][\mathrm{S}]$} & O-sulf & $\mathrm{O}$ on $\mathrm{O}-\mathrm{S}$ bond \\
\hline & {$[\mathrm{OX} 1]=[\mathrm{S}]$} & $\mathrm{O}=$ sulf & $\mathrm{O}$ on $\mathrm{O}=\mathrm{S}$ bond \\
\hline & {$[\mathrm{OX} 2 \mathrm{H} 2]$} & OW & O on Water \\
\hline & {$[\$([\mathrm{OH} 1][\mathrm{C}]=[\mathrm{O}])]$} & Ohacid & $\mathrm{O}$ of acid with $\mathrm{H}$ \\
\hline \multirow[t]{7}{*}{$\mathrm{S}$} & {$[\mathrm{S}]$} & $\mathrm{S}^{*}$ & wild matching $\mathrm{S}$ \\
\hline & {$[\mathrm{S}][\mathrm{C}]$} & Snonpol & $\mathrm{S}$ on non-polar $\mathrm{C}$ via single bond \\
\hline & {$[\mathrm{S}]=[\mathrm{C}]$} & Snonpol & $\mathrm{S}$ on non-polar $\mathrm{C}$ via double bond \\
\hline & {$[\mathrm{S}][\mathrm{N}]$} & Spolar & $\mathrm{S}$ connected to $\mathrm{N}$ \\
\hline & {$[\mathrm{S}][\mathrm{O}]$} & Spolar & $\mathrm{S}$ connected to $\mathrm{O}$ \\
\hline & {$[\mathrm{S}]=[\mathrm{O}]$} & Spolar & $\mathrm{S}$ connected to $\mathrm{O}$ via double bond \\
\hline & {$[\mathrm{s}]$} & Sar & $\mathrm{S}$ on aromatic system \\
\hline \multirow[t]{2}{*}{$P$} & {$[\mathrm{P}]$} & $\mathrm{P}^{*}$ & wild matching $\mathrm{P}$ \\
\hline & {$[\mathrm{P}](=[\mathrm{O}])(\mathrm{O})([\mathrm{O}])[\mathrm{O}]$} & Po4 & $\mathrm{P}$ on $\mathrm{PO} 4$ \\
\hline \multirow[t]{2}{*}{$\mathrm{F}$} & {$[\mathrm{F}][\mathrm{A}]$} & Fnonar & F connected to non-aromatic atom \\
\hline & {$[\mathrm{F}][\mathrm{a}]$} & Far & F connected to aromatic atom \\
\hline \multirow[t]{2}{*}{$\mathrm{Cl}$} & {$[\mathrm{Cl}][\mathrm{A}]$} & Clnonar & $\mathrm{Cl}$ connected to non-aromatic atom \\
\hline & {$[\mathrm{Cl}][\mathrm{a}]$} & Clar & $\mathrm{Cl}$ connected to aromatic atom \\
\hline \multirow[t]{2}{*}{$\mathrm{Br}$} & {$[\mathrm{Br}][\mathrm{A}]$} & Brnonar & Br connected to non-aromatic atom \\
\hline & {$[\mathrm{Br}][\mathrm{a}]$} & Brar & Br connected to aromatic atom \\
\hline \multirow[t]{2}{*}{$\mathrm{I}$} & {$[\mathrm{I}][\mathrm{A}]$} & Inonar & I connected to non-aromatic atom \\
\hline & {$[\mathrm{I}][\mathrm{a}]$} & Iar & I connected to aromatic atom \\
\hline
\end{tabular}


Table S3. Comparison of molecular polarizabilities calculated with LR-CCSD/aug-cc-pvdz (CCSD) and MP2/aug-cc-pvtz (MP2) at the same geometry for 52 representative molecules. Both the geometry and CCSD data are taken from reference. Polarizabilities are in $\AA^{3}$.

\begin{tabular}{llrrr}
\hline Index & Molecule & CCSD & MP2 & Diff. \\
\hline 1 & QM7B_0041.xyz & 5.06 & 5.03 & 0.02 \\
2 & QM7B_0067.xyz & 12.75 & 12.58 & 0.18 \\
3 & QM7B_0100.xyz & 12.54 & 12.52 & 0.02 \\
4 & QM7B_0131.xyz & 5.01 & 4.99 & 0.02 \\
5 & QM7B_0158.xyz & 9.65 & 9.60 & 0.05 \\
6 & QM7B_0191.xyz & 10.44 & 10.42 & 0.02 \\
7 & QM7B_0387.xyz & 9.15 & 8.98 & 0.18 \\
8 & QM7B_0396.xyz & 12.88 & 12.76 & 0.12 \\
9 & QM7B_0425.xyz & 8.88 & 8.73 & 0.15 \\
10 & QM7B_0474.xyz & 7.99 & 7.89 & 0.10 \\
11 & QM7B_0533.xyz & 10.44 & 10.35 & 0.09 \\
12 & QM7B_0616.xyz & 12.35 & 12.30 & 0.05 \\
13 & QM7B_0651.xyz & 11.05 & 11.02 & 0.03 \\
14 & QM7B_0679.xyz & 14.89 & 15.19 & -0.30 \\
15 & QM7B_0965.xyz & 11.25 & 11.25 & 0.00 \\
16 & QM7B_0967.xyz & 8.26 & 8.19 & 0.07 \\
17 & QM7B_1218.xyz & 10.44 & 10.32 & 0.12 \\
18 & QM7B_1314.xyz & 8.04 & 7.99 & 0.05 \\
19 & QM7B_1353.xyz & 9.82 & 9.78 & 0.05 \\
20 & QM7B_1385.xyz & 9.57 & 9.46 & 0.11 \\
21 & QM7B_1398.xyz & 9.03 & 8.91 & 0.11 \\
22 & QM7B_1460.xyz & 10.80 & 10.69 & 0.11 \\
23 & QM7B_1505.xyz & 9.97 & 9.89 & 0.08 \\
24 & QM7B_1597.xyz & 10.16 & 10.14 & 0.02 \\
25 & QM7B_1626.xyz & 9.25 & 9.14 & 0.11 \\
26 & QM7B_1679.xyz & 9.66 & 9.57 & 0.09 \\
27 & QM7B_1801.xyz & 10.90 & 10.89 & 0.01 \\
28 & QM7B_1828.xyz & 10.11 & 10.09 & 0.03 \\
29 & QM7B_1883.xyz & 9.24 & 9.23 & 0.01 \\
30 & QM7B_2079.xyz & 6.33 & 6.29 & 0.04 \\
31 & QM7B_2116.xyz & 10.04 & 9.97 & 0.07 \\
32 & QM7B_2250.xyz & 8.01 & 7.94 & 0.07 \\
33 & QM7B_2441.xyz & 8.73 & 8.68 & 0.05 \\
34 & QM7B_2515.xyz & 9.58 & 9.46 & 0.12 \\
35 & QM7B_2577.xyz & 9.77 & 9.76 & 0.00 \\
36 & QM7B_2910.xyz & 10.91 & 10.88 & 0.03 \\
37 & QM7B_3066.xyz & 9.45 & 9.40 & 0.05 \\
38 & QM7B_3109.xyz & 9.76 & 9.71 & 0.04 \\
40 & QM7B_3481.xyz & 10.12 & 10.10 & 0.02 \\
& QM7B_4044.xyz & 9.71 & 9.61 & 0.10
\end{tabular}




\begin{tabular}{llrrr}
41 & QM7B_4092.xyz & 10.30 & 10.26 & 0.04 \\
42 & QM7B_4212.xyz & 12.65 & 12.57 & 0.09 \\
43 & QM7B_4256.xyz & 9.35 & 9.30 & 0.05 \\
44 & QM7B_4387.xyz & 9.90 & 9.84 & 0.06 \\
45 & QM7B_4561.xyz & 9.81 & 9.71 & 0.10 \\
46 & QM7B_4828.xyz & 10.23 & 10.19 & 0.04 \\
47 & QM7B_4955.xyz & 10.50 & 10.50 & -0.01 \\
48 & QM7B_5334.xyz & 10.87 & 10.85 & 0.02 \\
49 & QM7B_5352.xyz & 9.95 & 9.92 & 0.03 \\
50 & QM7B_5397.xyz & 10.41 & 10.34 & 0.07 \\
51 & QM7B_6342.xyz & 12.71 & 12.70 & 0.01 \\
52 & QM7B_6800.xyz & 12.39 & 12.31 & 0.08 \\
\hline \multicolumn{4}{c}{ Mean signed difference } & $\mathbf{0 . 0 5}$ \\
\hline
\end{tabular}

a. The molecular geometry was taken from QM7b dataset without further optimization; CCSD data were taken from Wilkins et al. ${ }^{1}$ 
Table S4. Mean molecular polarizability of common gas molecules calculated from MP2/aug-cc-pvtz and AMOEBA model with the final set of parameters.

\begin{tabular}{cllll}
\hline Molecule & MP2 & Model & Diff. & \% Diff \\
\hline $\mathrm{CO}_{2}$ & 2.70 & 2.84 & 0.14 & 5.1 \\
$\mathrm{CO}$ & 3.09 & 2.85 & -0.24 & -7.7 \\
$\mathrm{H}_{2}$ & 0.77 & 0.75 & -0.02 & -2.1 \\
$\mathrm{~N}_{2}$ & 1.75 & 1.88 & 0.13 & 7.2 \\
$\mathrm{NO}_{2}$ & 2.66 & 2.62 & -0.04 & -1.5 \\
$\mathrm{O}_{2}$ & 1.39 & 1.43 & 0.04 & 3.2 \\
\hline
\end{tabular}


Table S5. Molecular polarizabilities (in $\AA^{3}$ ) from experiment and the improved AMOEBA model. Isotropic molecular polarizabilities are highlighted in bold.

\begin{tabular}{|c|c|c|c|c|c|c|}
\hline \multirow[b]{2}{*}{ Index } & \multirow[b]{2}{*}{ Molecule } & \multirow{2}{*}{$\begin{array}{c}\text { Expt. } \\
\alpha_{i s o}\end{array}$} & \multicolumn{4}{|c|}{ This work } \\
\hline & & & $\alpha_{x x}$ & $\alpha_{y y}$ & $\alpha_{z z}$ & $\alpha_{i s o}$ \\
\hline 1 & 3-methyltetrahydropyran & 11.71 & 10.21 & 11.32 & 12.43 & 11.32 \\
\hline 2 & chloromethyloxirane & 8.19 & 7.18 & 7.57 & 9.54 & 8.10 \\
\hline 3 & propylene carbonate & 8.55 & 7.65 & 8.87 & 10.47 & 9.00 \\
\hline 4 & 1,1,1,3,3,3-hexafluoro-2-propanol & 7.20 & 6.25 & 7.12 & 7.72 & 7.03 \\
\hline 5 & 1,1,1-trichloroethane & 10.44 & 9.50 & 10.04 & 10.04 & 9.86 \\
\hline 6 & 1,1,1-trichlorotrifluoroethane & 10.47 & 9.58 & 10.18 & 10.18 & 9.98 \\
\hline 7 & 1,1,2,2-tetrachloroethane & 12.21 & 9.31 & 13.08 & 13.25 & 11.88 \\
\hline 8 & 1,1,2-trichloro-1,2,2-trifluoro-ethane & 10.43 & 9.14 & 10.17 & 10.48 & 9.93 \\
\hline 9 & 1,1,2-trichloroethane & 10.33 & 8.21 & 10.07 & 11.81 & 10.03 \\
\hline 10 & 1,1,3,3-tetraethylurea & 20.17 & 17.27 & 19.93 & 23.72 & 20.30 \\
\hline 11 & 1,1,3,3-tetramethylurea & 12.86 & 10.53 & 13.12 & 15.65 & 13.10 \\
\hline 12 & 1,1-dichloroethylene & 8.36 & 5.91 & 8.63 & 8.91 & 7.82 \\
\hline 13 & 1,1-dichloroethane & 8.38 & 6.86 & 8.38 & 8.87 & 8.04 \\
\hline 14 & 1,10-dichlorodecane & 23.04 & 18.28 & 20.53 & 31.37 & 23.39 \\
\hline 15 & 1,2,3,4-tetrahydronaphthalene & 17.05 & 11.54 & 17.93 & 21.61 & 17.03 \\
\hline 16 & 1,2,3,4-tetramethylbenzene & 17.81 & 11.89 & 19.88 & 22.48 & 18.08 \\
\hline 17 & 1,2,3-propanetriol & 8.14 & 7.43 & 7.92 & 9.58 & 8.31 \\
\hline 18 & 1,2,3-trichloropropane & 12.09 & 10.38 & 11.96 & 13.21 & 11.85 \\
\hline 19 & 1,2,4-trichlorobenzene & 16.32 & 10.78 & 17.67 & 21.88 & 16.78 \\
\hline 20 & 1,2-butanediol & 9.32 & 8.05 & 8.91 & 11.09 & 9.35 \\
\hline 21 & 1,2-diaminoethane & 7.21 & 6.31 & 6.71 & 7.58 & 6.87 \\
\hline 22 & 1,2-dibromoethane & 10.75 & 8.73 & 9.22 & 14.15 & 10.70 \\
\hline 23 & 1,2-dibromopropane & 12.54 & 10.07 & 11.62 & 15.57 & 12.42 \\
\hline 24 & 1,2-dichloro-1,1,2,3,3,3-hexafluoropropane & 10.52 & 9.18 & 10.05 & 10.71 & 9.98 \\
\hline 25 & 1,2-dichlorobenzene & 14.30 & 9.40 & 16.21 & 17.95 & 14.52 \\
\hline 26 & 1,2-dichloroethane & 8.43 & 6.82 & 7.33 & 10.50 & 8.22 \\
\hline 27 & 1,2-difluorobenzene & 10.42 & 6.70 & 12.10 & 12.27 & 10.36 \\
\hline 28 & 1,2-dimethoxybenzene & 15.69 & 10.69 & 17.81 & 19.24 & 15.91 \\
\hline 29 & 1,2-dimethoxyethane & 9.60 & 8.09 & 8.36 & 13.38 & 9.94 \\
\hline 30 & 1,2-Ethanediol & 5.72 & 5.06 & 6.05 & 6.15 & 5.75 \\
\hline 31 & 1,3,5-trifluorobenzene & 10.24 & 6.70 & 12.26 & 12.26 & 10.40 \\
\hline 32 & 1,3-butanediol & 9.37 & 8.01 & 9.12 & 10.90 & 9.34 \\
\hline 33 & 1,3-dibromobenzene & 16.59 & 11.90 & 18.53 & 23.22 & $\mathbf{1 7 . 8 8}$ \\
\hline 34 & 1,3-dichlorobenzene & 14.38 & 9.50 & 15.76 & 18.66 & 14.64 \\
\hline 35 & 1,3-dichloropropane & 10.17 & 9.11 & 9.35 & 11.22 & 9.89 \\
\hline 36 & 1,3-Dimethyl-2-oxohexahydropyrimidine & 13.80 & 10.57 & 15.08 & 16.81 & 14.15 \\
\hline 37 & 1,3-dimethylimidazolidin-2-one & 12.14 & 9.24 & 12.70 & 15.09 & 12.34 \\
\hline 38 & 1,3-dioxolane & 6.79 & 5.99 & 6.80 & 7.93 & 6.91 \\
\hline
\end{tabular}




\begin{tabular}{|c|c|c|c|c|c|c|}
\hline 39 & 1,3-propanediol & 7.55 & 6.90 & 7.08 & 8.70 & 7.56 \\
\hline 40 & 1,4-butanediol & 9.35 & 7.97 & 8.59 & 11.83 & 9.46 \\
\hline 41 & 1,4-cyclohexadiene & 10.48 & 7.88 & 12.52 & 12.70 & 11.03 \\
\hline 42 & 1,4-dichlorobutane & 12.02 & 10.51 & 11.04 & 13.34 & 11.63 \\
\hline 43 & 1,4-difluorobenzene & 10.27 & 6.70 & 12.02 & 12.36 & 10.36 \\
\hline 44 & 1,4-dioxane & 8.63 & 7.38 & 8.77 & 10.09 & 8.75 \\
\hline 45 & 1,5-pentanediol & 11.20 & 9.96 & 10.43 & 12.83 & 11.07 \\
\hline 46 & 1,8-cineole & 18.10 & 16.86 & 17.03 & 19.01 & 17.64 \\
\hline 47 & 1-bromobutane & 11.26 & 9.37 & 9.90 & 13.91 & 11.06 \\
\hline 48 & 1-butanol & 8.79 & 7.38 & 7.99 & 10.56 & 8.64 \\
\hline 49 & 1-chloronaphthalene & 19.37 & 11.26 & 21.80 & 23.93 & 19.00 \\
\hline 50 & 1-decanol & 19.83 & 15.67 & 16.83 & 28.04 & 20.18 \\
\hline 51 & 1-heptanol & 14.30 & 11.55 & 12.40 & 19.08 & 14.34 \\
\hline 52 & 1-hexanol & 12.46 & 10.59 & 11.46 & 14.48 & 12.18 \\
\hline 53 & 1-hexene & 11.79 & 9.61 & 10.16 & 14.95 & 11.57 \\
\hline 54 & 1-hexyne & 11.09 & 9.07 & 9.93 & 14.88 & 11.29 \\
\hline 55 & 1-iodonaphthalene & 22.49 & 11.68 & 22.56 & 24.44 & 19.56 \\
\hline 56 & 1-iodopropane & 11.50 & 8.90 & 9.32 & 13.54 & 10.59 \\
\hline 57 & 1-methyl-2-pyrrolidinone & 10.66 & 8.46 & 11.25 & 12.55 & 10.76 \\
\hline 58 & 1-methylimidazole & 9.24 & 6.80 & 10.18 & 11.99 & 9.66 \\
\hline 59 & 1-methylpiperidine & 12.62 & 10.59 & 12.38 & 12.90 & 11.96 \\
\hline 60 & 1-methylnaphthalene & 19.42 & 11.23 & 21.43 & 23.69 & 18.79 \\
\hline 61 & 1-nitropropane & 8.60 & 7.64 & 8.03 & 8.90 & 8.19 \\
\hline 62 & 1-octanol & 16.14 & 12.92 & 13.89 & 22.02 & 16.28 \\
\hline 63 & 1-pentanol & 10.61 & 8.83 & 9.49 & 13.00 & 10.44 \\
\hline 64 & 1-phenylethanol & 14.62 & 10.18 & 15.57 & 18.89 & 14.88 \\
\hline 65 & 1-propanol & 6.96 & 5.98 & 6.41 & 8.02 & 6.80 \\
\hline 66 & 2,2,2-trichloroethanol & 11.00 & 10.15 & 10.49 & 11.18 & 10.61 \\
\hline 67 & 2,2,2-trifluoroethanol & 5.20 & 4.67 & 5.11 & 5.60 & 5.13 \\
\hline 68 & 2,2,3,3-tetrafluoro-1-propanol & 7.08 & 6.28 & 6.63 & 8.14 & 7.02 \\
\hline 69 & 2,2,4,4-tetramethyl-3-pentanone & 17.37 & 15.60 & 16.04 & 19.16 & 16.93 \\
\hline 70 & 2,2,5,5-tetramethyltetrahydrofuran & 15.40 & 13.65 & 14.51 & 16.71 & 14.96 \\
\hline 71 & 2,3,4,5,6-pentafluorotoluene & 12.24 & 8.09 & 13.67 & 15.75 & 12.50 \\
\hline 72 & 2,3-butanediol & 9.33 & 8.26 & 9.11 & 10.50 & 9.29 \\
\hline 73 & 2,4,6-collidine & 15.48 & 10.55 & 18.43 & 18.46 & 15.81 \\
\hline 74 & 2,4-dimethyl-3-pentanone & 13.70 & 12.07 & 12.86 & 15.81 & 13.58 \\
\hline 75 & 2,4-lutidine & 13.40 & 9.19 & 14.78 & 17.25 & 13.74 \\
\hline 76 & 2,4-pentanedione & 10.99 & 9.32 & 10.24 & 12.50 & 10.69 \\
\hline 77 & 2,5-dibromotoluene & 18.72 & 13.08 & 19.64 & 26.84 & 19.85 \\
\hline 78 & 2,6-di-tert-butylpyridine & 24.98 & 19.23 & 24.75 & 29.71 & 24.56 \\
\hline 79 & 2,6-difluoropyridine & 9.43 & 6.42 & 11.06 & 11.86 & 9.78 \\
\hline 80 & 2,6-dimethyl-4-heptanone & 17.50 & 14.81 & 16.17 & 20.73 & 17.23 \\
\hline 81 & 2,6-lutidine & 13.54 & 9.20 & 14.82 & 17.29 & 13.77 \\
\hline 82 & 2-(hydroxymethyl)furan & 9.84 & 7.46 & 10.34 & 12.83 & 10.21 \\
\hline
\end{tabular}




\begin{tabular}{|c|c|c|c|c|c|c|}
\hline 83 & 2-(n-butoxy)ethanol & 13.10 & 11.89 & 12.33 & 15.14 & 13.12 \\
\hline 84 & 2-(tert-butyl)phenol & 18.60 & 13.51 & 19.26 & 22.32 & 18.37 \\
\hline 85 & 2-ethoxyethanol & 9.48 & 8.07 & 8.63 & 12.46 & 9.72 \\
\hline 86 & 2-amino-1-butanol & 10.09 & 8.14 & 9.92 & 11.60 & 9.89 \\
\hline 87 & 2-aminoethanol & 6.48 & 5.69 & 6.18 & 7.08 & 6.31 \\
\hline 88 & 2-bromopyridine & 12.78 & 9.09 & 13.61 & 17.85 & 13.52 \\
\hline 89 & 2-butanol & 8.77 & 7.59 & 8.15 & 9.95 & 8.56 \\
\hline 90 & 2-butanone & 8.25 & 6.79 & 7.95 & 10.14 & 8.29 \\
\hline 91 & 2-chloroaniline & 14.05 & 8.71 & 15.42 & 17.15 & 13.76 \\
\hline 92 & 2-chloroethanol & 7.03 & 6.01 & 6.91 & 7.89 & 6.94 \\
\hline 93 & 2-chloropyridine & 11.60 & 7.86 & 12.47 & 15.38 & 11.90 \\
\hline 94 & 2-cyanoethanol & 6.92 & 6.13 & 6.56 & 9.09 & 7.26 \\
\hline 95 & 2-ethylphenol & 14.60 & 10.26 & 15.90 & 18.28 & 14.81 \\
\hline 96 & 2-hexanone & 11.95 & 9.57 & 10.93 & 15.73 & 12.07 \\
\hline 97 & 2-iodopropane & 11.67 & 8.89 & 10.22 & 12.24 & 10.45 \\
\hline 98 & 2-isopropylphenol & 16.40 & 11.84 & 17.65 & 20.44 & 16.64 \\
\hline 99 & 2-methoxyethanol & 7.62 & 6.57 & 7.37 & 9.35 & 7.76 \\
\hline 100 & 2-methoxyphenol & 13.60 & 8.88 & 15.04 & 17.81 & 13.91 \\
\hline 101 & 2-methyl-1-propanol & 8.81 & 7.34 & 8.76 & 9.45 & 8.52 \\
\hline 102 & 2-methyl-2-butanol & 10.64 & 9.35 & 10.00 & 11.45 & 10.27 \\
\hline 103 & 2-methyl-2-nitropropane & 10.30 & 9.44 & 10.12 & 10.23 & 9.93 \\
\hline 104 & 2-methylbutane & 10.13 & 8.26 & 9.52 & 10.85 & 9.54 \\
\hline 105 & 2-nitropropane & 8.59 & 7.62 & 8.17 & 8.84 & 8.21 \\
\hline 106 & 2-pentanol & 10.57 & 9.29 & 9.87 & 11.68 & 10.28 \\
\hline 107 & 2-pentanone & 10.11 & 8.18 & 9.48 & 12.86 & 10.17 \\
\hline 108 & 2-picoline & 11.57 & 7.83 & 12.43 & 14.94 & 11.73 \\
\hline 109 & allyl alcohol & 6.71 & 5.84 & 6.34 & 8.14 & 6.78 \\
\hline 110 & 2-propenenitrile & 6.24 & 4.85 & 6.09 & 8.13 & 6.36 \\
\hline 111 & 2-propyne-1-ol & 5.98 & 5.36 & 5.97 & 8.18 & 6.50 \\
\hline 112 & 3,3-dimethyl-2-butanone & 11.85 & 10.68 & 11.50 & 12.85 & 11.68 \\
\hline 113 & 3,4-lutidine & 13.34 & 9.09 & 15.16 & 16.46 & 13.57 \\
\hline 114 & 3-bromopyridine & 12.76 & 9.07 & 13.50 & 17.75 & 13.44 \\
\hline 115 & 3-chloro-1,1,1-trifluoropropane & 8.23 & 6.98 & 7.42 & 10.08 & 8.16 \\
\hline 116 & 3-chlorophenol & 13.60 & 8.44 & 14.70 & 16.55 & 13.23 \\
\hline 117 & 3-chloropropyne & 7.52 & 6.27 & 7.05 & 9.83 & 7.72 \\
\hline 118 & 3-ethyl-3-pentanol & 14.44 & 12.16 & 14.00 & 15.28 & 13.81 \\
\hline 119 & 3-heptanone & 13.70 & 11.53 & 12.23 & 17.91 & 13.89 \\
\hline 120 & 3-methoxypropanenitrile & 8.79 & 7.58 & 8.15 & 12.31 & 9.35 \\
\hline 121 & 3-methyl-1-butanol & 10.61 & 8.72 & 10.28 & 12.09 & 10.36 \\
\hline 122 & 3-methyl-2-butanone & 10.02 & 8.78 & 9.76 & 11.47 & 10.00 \\
\hline 123 & 3-methyl-2-oxazolidinone & 9.29 & 7.56 & 10.14 & 11.50 & 9.73 \\
\hline 124 & 3-pentanone & 10.03 & 8.17 & 9.44 & 12.87 & 10.16 \\
\hline 125 & 3-picoline & 11.50 & 7.82 & 12.34 & 14.92 & 11.69 \\
\hline 126 & 4-methylphenol & 13.20 & 8.41 & 13.63 & 17.20 & 13.08 \\
\hline
\end{tabular}




\begin{tabular}{|c|c|c|c|c|c|c|}
\hline 127 & 5-acetyl-5-methyl-1,3-dioxane & 14.26 & 12.56 & 13.58 & 17.00 & 14.38 \\
\hline 128 & 5-isopropyl-2-methylphenol & 18.60 & 13.67 & 18.26 & 24.37 & 18.77 \\
\hline 129 & 5-nonanone & 17.44 & 14.86 & 17.40 & 18.82 & 17.02 \\
\hline 130 & N,N,N',N'-tetraethylsulfamide & 21.32 & 19.09 & 21.70 & 24.45 & 21.74 \\
\hline 131 & tetramethylguanidine & 13.85 & 11.13 & 13.76 & 15.96 & 13.61 \\
\hline 132 & $\mathrm{~N}, \mathrm{~N}$-diethylformamide & 11.56 & 9.75 & 11.75 & 12.96 & 11.49 \\
\hline 133 & $\mathrm{~N}, \mathrm{~N}$-dimethylacetamide & 9.69 & 7.57 & 10.27 & 11.44 & 9.76 \\
\hline 134 & $\mathrm{~N}, \mathrm{~N}$-dimethylaniline & 16.29 & 10.67 & 16.25 & 19.99 & 15.64 \\
\hline 135 & $\mathrm{~N}, \mathrm{~N}$-dimethylbenzylamine & $\mathbf{1 7 . 5 3}$ & 13.38 & 16.65 & 22.74 & 17.59 \\
\hline 136 & N,N-dimethylcyclohexylamine & 16.09 & 13.45 & 15.91 & 17.25 & 15.53 \\
\hline 137 & $\mathrm{~N}, \mathrm{~N}$-dimethylformamide & 7.93 & 6.25 & 8.60 & 9.00 & 7.95 \\
\hline 138 & $\mathrm{~N}, \mathrm{~N}$-dimethylthioformamide & 11.39 & 7.83 & 10.09 & 12.34 & 10.09 \\
\hline 139 & $\mathrm{~N}$-methylacetamide & 7.85 & 6.20 & 7.59 & 9.93 & 7.91 \\
\hline 140 & N-methylaniline & 14.21 & 8.85 & 14.41 & 17.88 & 13.71 \\
\hline 141 & N-methylcyclohexylamine & 14.02 & 12.07 & 13.57 & 15.48 & 13.71 \\
\hline 142 & N-methylformamide & 6.01 & 4.78 & 6.09 & 7.27 & 6.05 \\
\hline 143 & N-methylformanilide & 15.89 & 10.99 & 16.37 & 20.79 & 16.05 \\
\hline 144 & acetic acid & 5.18 & 4.32 & 5.32 & 6.42 & 5.36 \\
\hline 145 & acetic anhydride & 8.93 & 7.61 & 9.00 & 11.83 & 9.48 \\
\hline 146 & acetone & 6.47 & 5.39 & 6.54 & 7.45 & 6.46 \\
\hline 147 & acetonitrile & 4.44 & 4.14 & 4.14 & 5.76 & 4.68 \\
\hline 148 & acetophenone & 14.45 & 9.30 & 15.41 & 18.98 & 14.56 \\
\hline 149 & allylamine & 7.57 & 6.27 & 6.89 & 8.84 & 7.33 \\
\hline 150 & aniline & 12.16 & 7.37 & 12.92 & 14.66 & 11.65 \\
\hline 151 & benzaldehyde & 12.70 & 7.99 & 13.72 & 16.25 & 12.65 \\
\hline 152 & benzene & 10.44 & 6.70 & 12.05 & 12.05 & 10.27 \\
\hline 153 & benzonitrile & 12.57 & 8.12 & 13.34 & 16.38 & 12.61 \\
\hline 154 & benzophenone & 22.50 & 15.47 & 22.13 & 31.60 & 23.07 \\
\hline 155 & benzoyl chloride & 14.70 & 9.19 & 15.51 & 19.10 & 14.60 \\
\hline 156 & benzyl alcohol & 12.89 & 8.91 & 13.79 & 16.39 & 13.03 \\
\hline 157 & biacetyl & 8.30 & 6.69 & 8.67 & 10.75 & 8.70 \\
\hline 158 & bis(2-methoxyethyl)ether & 13.90 & 11.51 & 12.17 & 20.98 & 14.89 \\
\hline 159 & bromobenzene & 13.52 & 9.32 & 14.38 & 18.37 & 14.03 \\
\hline 160 & bromoform & 11.87 & 9.53 & 12.73 & 12.73 & 11.67 \\
\hline 161 & bromotrichloromethane & 11.67 & 10.83 & 10.83 & 11.74 & 11.13 \\
\hline 162 & butanenitrile & 8.12 & 6.97 & 7.43 & 10.48 & 8.29 \\
\hline 163 & butyraldehyde & 8.20 & 6.83 & 7.87 & 10.20 & 8.30 \\
\hline 164 & carbon disulfide & 8.51 & 6.26 & 6.26 & 9.48 & 7.34 \\
\hline 165 & carbon tetrachloride & 10.54 & 9.95 & 9.95 & 9.95 & 9.95 \\
\hline 166 & chloroacetonitrile & 6.40 & 5.54 & 6.25 & 8.07 & 6.62 \\
\hline 167 & cinnamaldehyde & 17.50 & 10.18 & 16.47 & 23.33 & 16.66 \\
\hline 168 & cis-1,2-dichloroethylene & 8.08 & 5.92 & 8.13 & 9.28 & 7.78 \\
\hline 169 & cycloheptane & 12.84 & 10.47 & 12.91 & 13.21 & 12.20 \\
\hline 170 & cycloheptatriene & 12.46 & 9.22 & 13.78 & 14.12 & 12.37 \\
\hline
\end{tabular}




\begin{tabular}{|c|c|c|c|c|c|c|}
\hline 171 & cyclohexane & 11.04 & 9.06 & 11.16 & 11.16 & 10.46 \\
\hline 172 & cyclohexanol & 11.40 & 10.11 & 11.58 & 11.79 & 11.16 \\
\hline 173 & cyclohexanone & 11.12 & 9.15 & 11.68 & 11.89 & 10.91 \\
\hline 174 & cyclohexene & 10.79 & 8.40 & 11.56 & 11.57 & 10.51 \\
\hline 175 & cyclohexylamine & 12.47 & 10.65 & 11.92 & 12.59 & 11.72 \\
\hline 176 & cyclohexylbenzene & 20.77 & 16.19 & 18.91 & 27.07 & 20.72 \\
\hline 177 & cyclooctane & 14.62 & 12.02 & 14.78 & 14.95 & 13.92 \\
\hline 178 & cyclooctatetraene & 14.07 & 10.82 & 15.32 & 15.32 & 13.82 \\
\hline 179 & cyclopentane & 9.23 & 7.72 & 9.24 & 9.25 & 8.74 \\
\hline 180 & cyclopentanol & 9.72 & 8.32 & 9.67 & 10.55 & 9.51 \\
\hline 181 & cyclopropyl methyl ketone & 9.51 & 8.02 & 8.72 & 11.13 & 9.29 \\
\hline 182 & di-n-butyl ether & 16.31 & 14.54 & 14.78 & 18.82 & 16.05 \\
\hline 183 & di-n-butyl phthalate & 30.75 & 22.80 & 34.49 & 37.86 & 31.72 \\
\hline 184 & di-n-butylamine & 16.90 & 14.75 & 15.16 & 19.99 & 16.64 \\
\hline 185 & di-tert-butyl ether & 16.27 & 14.75 & 15.05 & 17.15 & 15.65 \\
\hline 186 & di-tert-butyl sulfide & 19.31 & 16.37 & 16.60 & 21.00 & 17.99 \\
\hline 187 & dibromomethane & 8.75 & 7.29 & 8.03 & 10.65 & 8.66 \\
\hline 188 & dichloroacetic acid & 9.10 & 8.35 & 9.16 & 9.71 & 9.07 \\
\hline 189 & dicyclopropyl ketone & 12.52 & 10.60 & 10.85 & 15.13 & 12.19 \\
\hline 190 & diethanolamine & 10.70 & 9.30 & 10.20 & 13.20 & 10.90 \\
\hline 191 & diethoxymethane & 11.40 & 9.74 & 11.01 & 13.61 & 11.45 \\
\hline 192 & diethyl carbonate & 11.32 & 9.98 & 10.24 & 15.40 & 11.87 \\
\hline 193 & diethyl disulfide & 14.59 & 12.11 & 12.56 & 17.04 & 13.90 \\
\hline 194 & diethyl malonate & 15.11 & 13.56 & 16.96 & 17.39 & 15.97 \\
\hline 195 & diethyl sulfate & 13.00 & 11.23 & 12.09 & 17.54 & 13.62 \\
\hline 196 & diethyl sulfide & 11.38 & 8.99 & 10.00 & 14.66 & 11.22 \\
\hline 197 & diethyl sulfite & 12.71 & 10.33 & 12.76 & 15.99 & 13.02 \\
\hline 198 & diethylamine & 9.63 & 7.82 & 8.53 & 11.92 & 9.43 \\
\hline 199 & diethylene glycol & 10.09 & 8.56 & 9.66 & 13.14 & 10.46 \\
\hline 200 & diethyleneglycol dimethyl ether & 13.97 & 11.51 & 12.17 & 20.98 & 14.89 \\
\hline 201 & diiodomethane & 12.96 & 9.44 & 10.25 & 14.11 & 11.27 \\
\hline 202 & diisopropyl ether & 12.65 & 10.74 & 12.14 & 14.13 & 12.34 \\
\hline 203 & diisopropylamine & 13.45 & 11.40 & 12.19 & 14.99 & 12.86 \\
\hline 204 & dimethoxymethane & 7.68 & 6.63 & 7.60 & 8.91 & 7.71 \\
\hline 205 & dimethyl carbonate & 7.56 & 6.43 & 7.19 & 11.14 & 8.25 \\
\hline 206 & dimethyl disulfide & 10.84 & 8.93 & 9.69 & 12.01 & 10.21 \\
\hline 207 & dimethyl phthalate & 19.59 & 13.72 & 22.78 & 25.55 & 20.68 \\
\hline 208 & dimethyl sulfate & 8.88 & 8.36 & 8.36 & 12.52 & 9.75 \\
\hline 209 & dimethyl sulfide & 7.63 & 6.29 & 7.30 & 8.73 & 7.44 \\
\hline 210 & dimethyl sulfite & 8.94 & 7.47 & 8.18 & 12.25 & 9.30 \\
\hline 211 & dimethyl sulfoxide & 8.03 & 6.57 & 7.79 & 8.49 & 7.62 \\
\hline 212 & diphenyl ether & 21.02 & 16.14 & 19.05 & 29.31 & 21.50 \\
\hline 213 & diphenylmethane & 21.90 & 18.84 & 19.59 & 28.66 & 22.36 \\
\hline 214 & ethanol & 5.13 & 4.53 & 4.99 & 5.50 & 5.01 \\
\hline
\end{tabular}




\begin{tabular}{|c|c|c|c|c|c|c|}
\hline 215 & ethyl acetate & 8.87 & 7.85 & 8.24 & 11.35 & 9.15 \\
\hline 216 & ethyl acetoacetate & 12.82 & 11.67 & 12.97 & 15.35 & 13.33 \\
\hline 217 & ethyl benzoate & 16.94 & 11.83 & 17.09 & 23.50 & 17.47 \\
\hline 218 & ethyl chloroacetate & 10.76 & 9.51 & 10.54 & 13.18 & 11.08 \\
\hline 219 & ethyl formate & 7.09 & 6.36 & 6.77 & 8.62 & 7.25 \\
\hline 220 & ethyl lactate & 11.34 & 9.98 & 11.00 & 14.07 & 11.68 \\
\hline 221 & ethyl propiolate & 10.08 & 8.69 & 9.14 & 14.20 & 10.67 \\
\hline 222 & Ethyl trichloroacetate & 14.82 & 13.50 & 13.82 & 17.27 & 14.86 \\
\hline 223 & ethyl vinyl ether & 8.74 & 6.87 & 7.99 & 11.61 & 8.82 \\
\hline 224 & ethylbenzene & 14.24 & 10.00 & 14.45 & 17.76 & 14.07 \\
\hline 225 & ethylene carbonate & 6.60 & 5.85 & 7.09 & 8.76 & 7.23 \\
\hline 226 & ethylene glycol dimethyl ether & 9.58 & 8.09 & 8.36 & 13.38 & 9.94 \\
\hline 227 & ethylene sulfite & 8.00 & 7.13 & 7.90 & 9.75 & 8.26 \\
\hline 228 & ethyl propanoate & 10.60 & 9.48 & 10.12 & 13.10 & 10.90 \\
\hline 229 & formamide & 4.22 & 3.20 & 4.27 & 4.92 & 4.13 \\
\hline 230 & formic acid & 3.39 & 2.86 & 3.69 & 4.13 & 3.56 \\
\hline 231 & furan & 7.35 & 5.23 & 8.12 & 9.03 & 7.46 \\
\hline 232 & gamma-butyrolactone & 7.94 & 6.79 & 8.33 & 9.49 & 8.20 \\
\hline 233 & hexachloro-1,3-butadiene & 19.75 & 16.54 & 17.61 & 21.95 & 18.70 \\
\hline 234 & hexachlorocyclopentadiene & 20.67 & 15.40 & 22.41 & 22.48 & 20.10 \\
\hline 235 & hexafluorobenzene & 10.56 & 6.72 & 12.48 & 12.48 & 10.56 \\
\hline 236 & hexamethylphosphoric acid triamide & 19.00 & 16.85 & 18.74 & 18.75 & 18.11 \\
\hline 237 & hexanenitrile & 11.84 & 9.90 & 10.47 & 15.56 & 11.98 \\
\hline 238 & iodoethane & 9.66 & 7.52 & 7.98 & 10.58 & 8.69 \\
\hline 239 & iodomethane & 7.68 & 6.19 & 6.19 & 8.31 & 6.90 \\
\hline 240 & isoamyl acetate & 14.48 & 13.31 & 13.44 & 16.59 & 14.45 \\
\hline 241 & isooctane & 15.63 & 13.28 & 14.35 & 16.78 & 14.80 \\
\hline 242 & isopropylbenzene & 16.10 & 12.02 & 15.66 & 19.94 & 15.87 \\
\hline 243 & m-xylene & 14.33 & 9.44 & 15.46 & 17.95 & 14.28 \\
\hline 244 & mesitylene & 16.25 & 10.79 & 19.11 & 19.11 & 16.33 \\
\hline 245 & methanesulfonic acid & 6.70 & 6.44 & 6.73 & 7.60 & 6.93 \\
\hline 246 & methyl acetate & 7.00 & 5.93 & 6.86 & 9.30 & 7.36 \\
\hline 247 & methyl acrylate & 8.85 & 6.63 & 9.10 & 11.65 & 9.13 \\
\hline 248 & methyl benzoate & 15.06 & 9.86 & 15.82 & 21.18 & 15.62 \\
\hline 249 & methyl butanoate & 10.67 & 9.24 & 9.96 & 13.74 & 10.98 \\
\hline 250 & methyl formate & 5.21 & 4.49 & 5.25 & 6.72 & 5.49 \\
\hline 251 & methyl hexanoate & 14.34 & 11.69 & 13.06 & 19.89 & 14.88 \\
\hline 252 & methyl methacrylate & 10.60 & 7.95 & 11.24 & 13.82 & 11.00 \\
\hline 253 & methyl octanoate & 18.09 & 14.56 & 16.43 & 25.20 & 18.73 \\
\hline 254 & methyl pentanoate & 12.53 & 10.11 & 11.34 & 17.71 & 13.05 \\
\hline 255 & methyl propionate & 8.82 & 7.33 & 8.37 & 11.97 & 9.22 \\
\hline 256 & methyl salicylate & 16.00 & 10.12 & 17.02 & 21.88 & 16.34 \\
\hline 257 & methyl trichloroacetate & 12.87 & 11.82 & 12.37 & 15.02 & 13.07 \\
\hline 258 & methyl trifluoroacetate & 7.19 & 6.07 & 7.00 & 9.31 & 7.46 \\
\hline
\end{tabular}




\begin{tabular}{|c|c|c|c|c|c|c|}
\hline 259 & hexamethyl phosphoramide & 16.05 & 16.85 & 18.74 & 18.75 & 18.11 \\
\hline 260 & morpholine & 9.40 & 8.03 & 9.24 & 10.52 & 9.26 \\
\hline 261 & morpholine-4-carbonitrile & 11.22 & 10.06 & 11.92 & 12.33 & 11.44 \\
\hline 262 & n-butanoic acid & 8.80 & 7.14 & 8.35 & 11.61 & 9.03 \\
\hline 263 & n-butyl acetate & 12.57 & 11.10 & 12.05 & 15.10 & 12.75 \\
\hline 264 & n-butyl methyl ether & 10.80 & 9.25 & 10.17 & 12.17 & 10.53 \\
\hline 265 & n-butylamine & 9.64 & 7.97 & 8.38 & 11.27 & 9.21 \\
\hline 266 & n-decane & 19.33 & 16.40 & 17.63 & 22.33 & 18.79 \\
\hline 267 & n-dodecane & 23.03 & 19.57 & 20.88 & 26.76 & 22.40 \\
\hline 268 & n-dodecanol & 23.50 & 18.42 & 19.75 & 34.15 & 24.11 \\
\hline 269 & n-heptane & 13.81 & 11.19 & 11.99 & 16.97 & 13.38 \\
\hline 270 & n-hexadecane & 30.42 & 26.09 & 28.44 & 36.39 & 30.31 \\
\hline 271 & n-octane & 15.60 & 13.06 & 13.68 & 18.66 & 15.13 \\
\hline 272 & n-pentane & 10.11 & 8.26 & 8.78 & 12.04 & 9.69 \\
\hline 273 & n-pentanoic acid & 10.60 & 8.53 & 9.88 & 14.34 & 10.92 \\
\hline 274 & n-propyl acetate & 10.72 & 9.46 & 10.25 & 12.98 & 10.90 \\
\hline 275 & n-propyl formate & 8.93 & 8.09 & 8.52 & 10.43 & 9.01 \\
\hline 276 & n-undecane & 21.14 & 18.25 & 19.04 & 24.26 & 20.52 \\
\hline 277 & nitrobenzene & 13.00 & 7.88 & 13.84 & 16.16 & 12.63 \\
\hline 278 & nitrocyclohexane & 13.26 & 11.41 & 13.09 & 13.34 & 12.61 \\
\hline 279 & nitroethane & 6.76 & 5.40 & 6.46 & 7.59 & 6.48 \\
\hline 280 & nitromethane & 4.97 & 3.93 & 4.85 & 5.31 & 4.70 \\
\hline 281 & o-xylene & 14.25 & 9.34 & 15.74 & 17.38 & 14.15 \\
\hline 282 & p-chloroacetophenone & 16.50 & 10.69 & 16.67 & 23.16 & 16.84 \\
\hline 283 & p-methoxybenzaldehyde & 15.90 & 9.84 & 15.58 & 21.50 & 15.64 \\
\hline 284 & p-methylacetophenone & 16.40 & 10.66 & 16.61 & 22.73 & 16.67 \\
\hline 285 & pentachloroethane & 14.17 & 12.32 & 14.30 & 14.34 & 13.65 \\
\hline 286 & pentafluorobenzene & 10.43 & 6.71 & 12.31 & 12.50 & 10.51 \\
\hline 287 & pentafluoropyridine & 10.18 & 6.43 & 11.27 & 12.10 & 9.93 \\
\hline 288 & pentanenitrile & 9.99 & 8.51 & 9.72 & 11.94 & 10.06 \\
\hline 289 & perfluoro(methylcyclohexane) & 13.71 & 11.16 & 13.29 & 14.91 & $\mathbf{1 3 . 1 2}$ \\
\hline 290 & perfluoro-n-heptane & 14.70 & 12.10 & 12.29 & 18.46 & 14.29 \\
\hline 291 & perfluoro-n-hexane & 12.71 & 10.56 & 10.75 & 15.47 & 12.26 \\
\hline 292 & perfluoro-n-octane & 11.00 & 7.04 & 12.42 & 13.71 & 11.05 \\
\hline 293 & perfluorodecalin & 13.91 & 8.83 & 13.98 & 18.64 & 13.82 \\
\hline 294 & phenol & 15.80 & 12.47 & 16.49 & 19.88 & 16.28 \\
\hline 295 & phenylacethylene & 13.96 & 9.89 & 15.06 & 18.84 & 14.60 \\
\hline 296 & phenylacetone & 10.65 & 8.83 & 10.46 & 11.06 & 10.12 \\
\hline 297 & propanenitrile & 6.30 & 5.57 & 6.04 & 7.75 & 6.45 \\
\hline 298 & propanoic acid & 6.72 & 5.74 & 6.90 & 8.85 & 7.16 \\
\hline 299 & propargylamine & 7.29 & 5.99 & 6.48 & 8.69 & 7.05 \\
\hline 300 & propionaldehyde & 6.40 & 5.42 & 6.48 & 7.47 & 6.46 \\
\hline 301 & propiophenone & 16.20 & 10.67 & 16.67 & 22.40 & 16.58 \\
\hline 302 & pyridine & 9.58 & 6.44 & 11.05 & 11.66 & 9.72 \\
\hline
\end{tabular}




\begin{tabular}{|c|c|c|c|c|c|c|}
\hline 303 & pyrimidine & 8.59 & 6.17 & 10.39 & 10.93 & 9.17 \\
\hline 304 & pyrrole & 8.23 & 5.58 & 9.40 & 9.54 & 8.17 \\
\hline 305 & pyrrolidin-2-one & 8.66 & 7.02 & 9.33 & 9.96 & 8.77 \\
\hline 306 & pyrrolidine & 8.77 & 7.15 & 8.97 & 9.15 & 8.42 \\
\hline 307 & pyrrolidine-1-carbonitrile & 11.09 & 8.65 & 10.59 & 12.91 & 10.72 \\
\hline 308 & quinoline & 16.65 & 9.72 & 17.41 & 21.71 & 16.28 \\
\hline 309 & styrene & 14.50 & 8.97 & 14.68 & 18.65 & 14.10 \\
\hline 310 & sulfolane & 10.73 & 9.67 & 10.97 & 11.83 & 10.83 \\
\hline 311 & tert-butyl alcohol & 8.82 & 8.07 & 8.61 & 8.82 & 8.50 \\
\hline 312 & tert-amyl methyl ether & 12.48 & 11.06 & 12.66 & 12.81 & 12.17 \\
\hline 313 & tert-butyl ethyl ether & 12.62 & 11.39 & 11.52 & 14.04 & 12.32 \\
\hline 314 & tert-butyl methyl ether & 10.65 & 9.80 & 10.06 & 11.41 & 10.42 \\
\hline 315 & tert-butylamine & 9.69 & 8.76 & 9.10 & 9.24 & 9.03 \\
\hline 316 & tert-butyl hydroperoxide & 9.44 & 8.73 & 9.20 & 10.02 & 9.32 \\
\hline 317 & tetraethylene glycol & 18.71 & 15.57 & 19.29 & 23.91 & 19.59 \\
\hline 318 & tetrahydrofuran & 7.97 & 6.93 & 7.95 & 8.73 & 7.87 \\
\hline 319 & tetrahydrothiophene & 10.41 & 8.48 & 10.89 & 11.03 & 10.13 \\
\hline 320 & tetramethylene sulfoxide & 10.77 & 9.15 & 10.68 & 11.11 & 10.31 \\
\hline 321 & tetrahydropyran & 9.85 & 8.24 & 9.96 & 10.61 & 9.61 \\
\hline 322 & thiophene & 9.72 & 6.54 & 10.75 & 10.80 & 9.36 \\
\hline 323 & toluene & 12.40 & 8.08 & 13.23 & 15.47 & 12.26 \\
\hline 324 & tri-n-butyl phosphate & 27.55 & 23.01 & 27.52 & 30.89 & 27.14 \\
\hline 325 & tri-n-butylamine & 24.43 & 20.24 & 23.47 & 27.55 & 23.75 \\
\hline 326 & tri-n-propyl phosphate & 22.24 & 18.10 & 22.21 & 24.97 & 21.76 \\
\hline 327 & trichloroacetonitrile & 10.48 & 10.14 & 10.14 & 10.58 & 10.29 \\
\hline 328 & trichloroethylene & 10.08 & 7.14 & 10.03 & 11.78 & 9.65 \\
\hline 329 & triethanolamine & 15.10 & 13.38 & 15.35 & 16.84 & 15.19 \\
\hline 330 & triethylamine & 13.48 & 10.96 & 13.36 & 14.49 & 12.94 \\
\hline 331 & trimethyl orthoacetate & 11.91 & 10.06 & 12.42 & 13.87 & 12.12 \\
\hline 332 & trimethyl orthoformate & 10.09 & 8.70 & 10.79 & 11.63 & 10.37 \\
\hline 333 & trimethyl phosphate & 11.07 & 9.53 & 11.82 & 11.83 & 11.06 \\
\hline 334 & trimethylacetonitrile & 10.00 & 9.59 & 9.59 & 10.56 & 9.92 \\
\hline 335 & trimethylene sulfide & 8.64 & 7.20 & 8.86 & 9.13 & 8.40 \\
\hline 336 & vinyl acetate & 8.87 & 6.67 & 8.65 & 12.19 & 9.17 \\
\hline 337 & 2-methylphenol & 13.00 & 8.38 & 14.69 & 15.97 & 13.01 \\
\hline 338 & methyloxirane & 6.25 & 5.39 & 5.83 & 7.16 & 6.13 \\
\hline 339 & (trifluoromethyl)benzene & 12.25 & 8.18 & 13.36 & 15.44 & 12.33 \\
\hline 340 & 1,2-dibromobenzene & 16.43 & 11.62 & 19.62 & 21.57 & 17.60 \\
\hline 341 & 1,2-propanediol & 7.55 & 6.97 & 7.45 & 8.11 & 7.51 \\
\hline 342 & 1,3-difluorobenzene & 10.33 & 6.70 & 12.10 & 12.28 & 10.36 \\
\hline 343 & 1,5-cyclooctadiene & 14.16 & 11.76 & 14.55 & 15.70 & 14.00 \\
\hline 344 & 1-amino-2-propanol & 8.36 & 7.51 & 7.71 & 8.95 & 8.06 \\
\hline 345 & 1-bromopropane & 9.42 & 7.81 & 8.25 & 11.73 & 9.26 \\
\hline 346 & 1-chlorobutane & 10.12 & 8.41 & 9.29 & 11.71 & 9.81 \\
\hline
\end{tabular}




\begin{tabular}{|c|c|c|c|c|c|c|}
\hline 347 & 1-chloropropane & 8.32 & 6.99 & 7.84 & 9.03 & 7.95 \\
\hline 348 & 1-iodobutane & 13.33 & 10.42 & 10.97 & 15.79 & 12.39 \\
\hline 349 & 1-methylpyrrole & 10.24 & 7.07 & 10.83 & 12.77 & 10.22 \\
\hline 350 & 1-nonyne & 16.62 & 14.07 & 16.20 & 19.67 & 16.65 \\
\hline 351 & 2,2-dichloropropane & 10.32 & 9.44 & 9.73 & 10.14 & 9.77 \\
\hline 352 & 2,4-dimethyl-3-pentanol & 14.21 & 12.42 & 13.26 & 15.82 & 13.83 \\
\hline 353 & 2,4-dimethylphenol & 14.80 & 9.75 & 16.33 & 19.14 & 15.07 \\
\hline 354 & 2,5-dimethyltetrahydrofuran & 11.40 & 9.75 & 11.21 & 13.78 & 11.58 \\
\hline 355 & 2-cyanopyridine & 11.70 & 7.87 & 12.53 & 15.87 & 12.09 \\
\hline 356 & 2-fluoropyridine & 10.61 & 6.43 & 11.04 & 11.77 & 9.75 \\
\hline 357 & 2-methoxy-1,3-dioxolane & 9.36 & 8.41 & 9.03 & 10.85 & 9.43 \\
\hline 358 & 2-methyltetrahydrofuran & 9.95 & 8.34 & 9.42 & 11.43 & 9.73 \\
\hline 359 & 2-propanol & 6.98 & 6.19 & 6.79 & 7.29 & 6.76 \\
\hline 360 & 3-methylphenol & 13.00 & 8.41 & 14.62 & 16.13 & 13.05 \\
\hline 361 & 3-methylsulfolane & 12.65 & 11.56 & 12.35 & 13.72 & 12.54 \\
\hline 362 & 3-pentanol & 10.62 & 9.05 & 9.53 & 12.65 & 10.41 \\
\hline 363 & 3-phenyl-1-propanol & 16.44 & 12.79 & 16.62 & 19.98 & 16.46 \\
\hline 364 & 4-heptanone & 13.67 & 11.90 & 13.16 & 15.48 & 13.51 \\
\hline 365 & 4-methyl-2-pentanone & 11.98 & 10.04 & 11.24 & 14.37 & 11.88 \\
\hline 366 & 4-picoline & 11.52 & 7.82 & 12.84 & 14.39 & 11.68 \\
\hline 367 & N,N-diethylacetamide & 13.28 & 11.05 & 13.96 & 14.88 & 13.30 \\
\hline 368 & N,N-diethylcyanamide & 11.71 & 9.40 & 11.92 & 13.25 & 11.53 \\
\hline 369 & N,N-diisopropylcyanamide & 15.31 & 13.65 & 14.45 & 16.71 & 14.94 \\
\hline 370 & N,N-dimethylcyanamide & 7.94 & 6.38 & 8.27 & 9.05 & 7.90 \\
\hline 371 & $\mathrm{~N}, \mathrm{~N}$-dimethylpropionamide & 11.46 & 8.94 & 11.68 & 14.38 & 11.66 \\
\hline 372 & $\mathrm{~N}$-(tert-butyl)benzylamine & 21.11 & 15.99 & 20.45 & 27.33 & 21.26 \\
\hline 373 & N-benzylmethylamine & 15.52 & 10.86 & 15.47 & 21.09 & 15.81 \\
\hline 374 & benzoyl bromide & 15.70 & 10.12 & 16.51 & 21.03 & 15.89 \\
\hline 375 & benzylamine & 13.60 & 9.67 & 14.18 & 16.82 & 13.56 \\
\hline 376 & bis(2-chloroethyl)ether & 12.63 & 10.25 & 11.14 & 17.96 & 13.12 \\
\hline 377 & bromoethane & 7.60 & 6.42 & 6.89 & 8.88 & 7.40 \\
\hline 378 & chlorobenzene & 12.40 & 8.11 & 13.29 & 15.91 & 12.43 \\
\hline 379 & chlorocyclohexane & 13.03 & 11.35 & 12.43 & 13.32 & 12.37 \\
\hline 380 & chloroform & 8.53 & 6.81 & 8.77 & 8.77 & 8.12 \\
\hline 381 & cis-decalin & 17.40 & 13.59 & 17.09 & 20.11 & 16.93 \\
\hline 382 & cyclopentanone & 9.25 & 7.66 & 9.73 & 10.16 & 9.18 \\
\hline 383 & bis(2-chloroethyl)ether & 12.60 & 10.25 & 11.14 & 17.96 & 13.12 \\
\hline 384 & di-n-butyl sulfide & 18.74 & 15.83 & 17.34 & 21.14 & 18.10 \\
\hline 385 & di-n-propyl ether & 12.67 & 11.09 & 11.53 & 14.66 & 12.43 \\
\hline 386 & diallylamine & 12.84 & 10.79 & 11.37 & 17.42 & 13.19 \\
\hline 387 & dibenzyl ether & 24.50 & 17.16 & 26.06 & 33.76 & 25.66 \\
\hline 388 & dichloromethane & 6.52 & 5.37 & 5.94 & 7.51 & 6.27 \\
\hline 389 & diethyl ether & 8.98 & 7.51 & 8.03 & 11.15 & 8.90 \\
\hline 390 & Diethylene glycol diethyl ether & 17.67 & 14.57 & 15.46 & 26.05 & 18.69 \\
\hline
\end{tabular}




\begin{tabular}{|c|c|c|c|c|c|c|}
\hline 391 & diisopropyl sulfide & 15.19 & 12.44 & 13.71 & 17.74 & 14.63 \\
\hline 392 & dodecanenitrile & 22.68 & 18.00 & 19.40 & 33.84 & 23.75 \\
\hline 393 & ethyl phenyl ether & 15.02 & 9.99 & 15.19 & 20.41 & 15.20 \\
\hline 394 & ethyl salicylate & 17.80 & 12.09 & 18.28 & 24.21 & 18.19 \\
\hline 395 & fluorobenzene & 10.33 & 6.70 & 12.03 & 12.20 & 10.31 \\
\hline 396 & fluorotrichloromethane & 8.61 & 6.82 & 8.72 & 8.72 & 8.09 \\
\hline 397 & glyceryl triacetate & 19.31 & 17.18 & 20.97 & 24.18 & 20.78 \\
\hline 398 & hexachloropropene & 17.84 & 14.00 & 17.99 & 19.47 & 17.15 \\
\hline 399 & iodobenzene & 15.58 & 8.52 & 13.63 & 16.80 & 12.98 \\
\hline 400 & isopropyl nitrate & 9.61 & 7.72 & 8.85 & 10.59 & 9.05 \\
\hline 401 & methanol & 3.26 & 2.98 & 3.09 & 3.70 & 3.25 \\
\hline 402 & methyl decanoate & 21.73 & 17.37 & 19.68 & 30.85 & 22.63 \\
\hline 403 & methyl dodecanoate & 25.40 & 20.17 & 22.82 & 36.71 & 26.57 \\
\hline 404 & methyl phenyl ether & 13.11 & 8.57 & 13.72 & 17.20 & 13.16 \\
\hline 405 & methyl phenyl sulfide & 15.63 & 11.45 & 15.07 & 19.44 & 15.32 \\
\hline 406 & methylcyclohexane & 12.96 & 10.34 & 12.45 & 14.11 & 12.30 \\
\hline 407 & n-heptanoic acid & 14.30 & 11.66 & 13.28 & 18.93 & 14.62 \\
\hline 408 & n-hexane & 11.94 & 9.79 & 10.62 & 14.02 & 11.47 \\
\hline 409 & n-hexanoic acid & 12.50 & 10.14 & 11.48 & 16.58 & 12.73 \\
\hline 410 & n-nonane & 17.45 & 15.04 & 15.24 & 20.49 & 16.92 \\
\hline 411 & n-pentadecane & 28.55 & 23.16 & 26.95 & 35.67 & 28.59 \\
\hline 412 & p-xylene & 14.35 & 9.44 & 14.40 & 19.11 & 14.32 \\
\hline 413 & Pentamethylene sulfide & 12.30 & 9.90 & 12.81 & 12.85 & 11.85 \\
\hline 414 & piperidine-1-carbonitrile & 12.83 & 9.94 & 12.48 & 15.03 & 12.48 \\
\hline 415 & tetrachloroethylene & 12.07 & 8.28 & 13.07 & 13.09 & 11.48 \\
\hline 416 & 2,2'-Thiobis(ethanol) & 12.50 & 10.85 & 11.11 & 16.15 & 12.70 \\
\hline 417 & trans-1,2-dichloroethylene & 8.22 & 5.99 & 7.15 & 10.48 & 7.87 \\
\hline 418 & triethyl phosphate & 16.57 & 14.61 & 16.64 & 18.73 & 16.66 \\
\hline 419 & triethylene glycol & 14.36 & 12.68 & 13.77 & 18.84 & 15.09 \\
\hline 420 & Triethylene glycol dimethyl ether & 18.34 & 15.55 & 16.69 & 25.44 & 19.22 \\
\hline 421 & trifluoroacetic acid & 5.40 & 4.45 & 5.90 & 5.92 & 5.42 \\
\hline 422 & undecanenitrile & 20.94 & 16.63 & 17.98 & 30.74 & 21.78 \\
\hline
\end{tabular}


Table S6. Three-body energy calculated by AMOEBA model with two sets of atomic polarizabilities for the 3B-69 dataset. The $\operatorname{CCSD}(\mathrm{T}) / \mathrm{CBS}$ data was taken from literature. ${ }^{2}$

\begin{tabular}{|c|c|c|c|}
\hline Trimer cluster & $\operatorname{CCSD}(\mathrm{T}) / \mathrm{CBS}$ & $\begin{array}{l}\text { AMOEBA } \\
\text { (old polar) }\end{array}$ & $\begin{array}{l}\text { AMOEBA } \\
\text { (new polar) }\end{array}$ \\
\hline 01a-water & -1.39 & -1.55 & -1.53 \\
\hline 01b-water & 1.08 & 1.19 & 1.23 \\
\hline 01c-water & -2.42 & -2.87 & -2.77 \\
\hline 02a-formaldehyde & -0.18 & -0.16 & -0.19 \\
\hline 02b-formaldehyde & 0.18 & 0.17 & 0.20 \\
\hline 02c-formaldehyde & -0.09 & -0.04 & -0.05 \\
\hline 03a-methanol_ethyne & -1.31 & -1.46 & -1.39 \\
\hline 03b-methanol_ethyne & 0.05 & 0.03 & 0.03 \\
\hline 03c-methanol_ethyne & 0.02 & -0.13 & -0.13 \\
\hline 04a-acetonitrile & 0.25 & 0.17 & 0.19 \\
\hline 04b-acetonitrile & 0.34 & 0.26 & 0.29 \\
\hline 04c-acetonitrile & -0.17 & -0.15 & -0.21 \\
\hline 05a-nitromethane & -0.12 & -0.18 & -0.20 \\
\hline 05b-nitromethane & 0.26 & 0.17 & 0.18 \\
\hline 05c-nitromethane & 0.22 & 0.22 & 0.24 \\
\hline 06a-acetic_acid & 0.54 & 0.30 & 0.37 \\
\hline 06b-acetic_acid & -0.92 & -0.72 & -0.84 \\
\hline 06c-acetic_acid & -0.21 & -0.14 & -0.16 \\
\hline 07a-oxalic_acid & -0.22 & -0.16 & -0.20 \\
\hline 07b-oxalic_acid & -1.20 & -0.86 & -0.98 \\
\hline 07c-oxalic_acid & -0.78 & -0.53 & -0.61 \\
\hline 08a-vinylene_carbonate & -0.31 & -0.35 & -0.34 \\
\hline 08b-vinylene_carbonate & -0.15 & -0.23 & -0.22 \\
\hline 08c-vinylene_carbonate & -0.49 & -0.61 & -0.60 \\
\hline 09a-acetamide & -0.09 & -0.09 & -0.11 \\
\hline 09b-acetamide & 0.58 & 0.80 & 0.75 \\
\hline 09c-acetamide & -0.86 & -0.65 & -0.70 \\
\hline 10a-imidazole & -0.66 & -0.82 & -0.93 \\
\hline 10b-imidazole & 0.27 & 0.21 & 0.21 \\
\hline 10c-imidazole & -1.64 & -1.87 & -2.18 \\
\hline 11a-isoxazole & 0.18 & -0.08 & -0.07 \\
\hline 11b-isoxazole & 0.19 & -0.13 & -0.04 \\
\hline 11c-isoxazole & -0.13 & -0.05 & -0.06 \\
\hline 12a-pyrazole & -1.28 & -1.72 & -1.79 \\
\hline 12b-pyrazole & 0.07 & -0.03 & -0.02 \\
\hline 12c-pyrazole & -0.29 & -0.40 & -0.45 \\
\hline 13a-triazine & -0.01 & 0.00 & 0.00 \\
\hline 13b-triazine & 0.14 & 0.11 & 0.05 \\
\hline 13c-triazine & 0.13 & 0.02 & 0.03 \\
\hline 14a-cyanoacetamide & 1.46 & 0.03 & 0.11 \\
\hline 14b-cyanoacetamide & 0.35 & -0.43 & -0.48 \\
\hline
\end{tabular}




\begin{tabular}{|c|c|c|c|}
\hline 14c-cyanoacetamide & -1.23 & -0.81 & -1.03 \\
\hline 15a-cyanoguanidine & -0.91 & -0.10 & -0.50 \\
\hline 15b-cyanoguanidine & 0.58 & 0.33 & 0.42 \\
\hline 15c-cyanoguanidine & 0.14 & 0.19 & 0.19 \\
\hline 16a-triazolidinedione & -0.14 & -0.07 & -0.07 \\
\hline 16b-triazolidinedione & 0.78 & 1.05 & 1.01 \\
\hline 16c-triazolidinedione & 0.51 & 0.29 & 0.28 \\
\hline 17a-oxazolidinone & 0.54 & 0.46 & 0.47 \\
\hline 17b-oxazolidinone & -0.21 & -0.36 & -0.42 \\
\hline 17c-oxazolidinone & 0.15 & 0.12 & 0.13 \\
\hline 18a-succinic_anhydride & 0.43 & 0.20 & 0.23 \\
\hline 18b-succinic_anhydride & 0.00 & 0.01 & 0.00 \\
\hline 18c-succinic_anhydride & -0.13 & -0.16 & -0.19 \\
\hline 19a-benzene & 0.05 & -0.08 & -0.06 \\
\hline 19b-benzene & 0.15 & 0.08 & 0.07 \\
\hline 19c-benzene & -0.03 & -0.08 & -0.08 \\
\hline 20a-maleic_acid & 1.69 & 1.11 & 1.27 \\
\hline 20b-maleic_acid & -1.45 & -0.92 & -1.19 \\
\hline 20c-maleic_acid & -0.36 & -0.19 & -0.23 \\
\hline 21a-p-benzoquinone & 0.33 & 0.18 & 0.25 \\
\hline 21b-p-benzoquinone & 0.00 & -0.04 & -0.09 \\
\hline 21c-p-benzoquinone & 0.10 & 0.04 & 0.05 \\
\hline 22a-uracil & 0.00 & 0.56 & 0.41 \\
\hline 22b-uracil & -0.24 & -0.12 & -0.13 \\
\hline 22c-uracil & 0.02 & -0.01 & 0.01 \\
\hline 23a-cyclobutylfuran & 0.08 & -0.05 & -0.05 \\
\hline 23b-cyclobutylfuran & 0.14 & 0.06 & 0.06 \\
\hline 23c-cyclobutylfuran & 0.15 & 0.03 & 0.03 \\
\hline RMSE (kcal/mol) & $\mathbf{0}$ & 0.30 & 0.26 \\
\hline
\end{tabular}


Table S7. Atomic polarizability parameters (in $\AA^{3}$ ) for charged molecules. Parameters are derived by fitting polarizability only (fit-polar) and fitting electrostatic difference only (fit-esp). The final set of parameters are the averages of fit-polar and fit-esp. The atom types for charged molecules are illustrated in Figure S1.

\begin{tabular}{lllll}
\hline Index & Atom type & fit-polar & fit-esp & final \\
\hline 1 & C(S-) & 2.46896 & 3.08732 & 2.77814 \\
2 & C+ & 1.51746 & 1.54505 & 1.53125 \\
3 & Car- & 2.71847 & 2.20922 & 2.46385 \\
4 & Cn+ & 0.82718 & 0.78690 & 0.80704 \\
5 & Co- & 2.82278 & 2.85413 & 2.83845 \\
6 & Coo- & 1.86150 & 2.07823 & 1.96987 \\
7 & Cooo- & 2.43886 & 1.95324 & 2.19605 \\
8 & Hn+ & 0.25500 & 0.26853 & 0.26176 \\
9 & Hop & 0.36037 & 0.42908 & 0.39472 \\
10 & NX4+ & 0.74624 & 0.80870 & 0.77747 \\
11 & Nar- & 1.94962 & 2.02092 & 1.98527 \\
12 & Nc+ & 0.87828 & 0.85571 & 0.86700 \\
13 & O(C)P & 1.18271 & 1.23862 & 1.21067 \\
14 & O(H)P & 0.71154 & 0.87051 & 0.79102 \\
15 & O- & 3.28997 & 3.03702 & 3.16350 \\
16 & Oc- & 1.92556 & 1.93713 & 1.93135 \\
17 & Oc-2 & 2.79251 & 3.07775 & 2.93513 \\
18 & Oo- & 2.22656 & 2.02176 & 2.12416 \\
19 & Op- & 2.41788 & 2.28682 & 2.35235 \\
20 & Os- & 1.64427 & 1.51598 & 1.58013 \\
21 & Po4 & 1.43530 & 1.42792 & 1.43161 \\
22 & S- & 7.36477 & 5.66354 & 6.51416 \\
23 & So3- & 2.50520 & 2.43678 & 2.47099 \\
\hline & & & &
\end{tabular}


Table S8. Performance of the final parameters on the ESP and molecular polarizability sets for charged molecules. RMSE (in $\mathrm{kcal} / \mathrm{mol} / \mathrm{e}$ and $\AA^{3}$ for ESP and polarizability respectively) and unsigned mean percentage error (UMPE, defined in Eq.3 in the main text) are reported.

\begin{tabular}{|c|c|c|c|c|}
\hline \multirow{2}{*}{$\begin{array}{c}\text { Parameters } \\
\text { fit-polar }\end{array}$} & \multicolumn{2}{|c|}{$\begin{array}{c}\text { ESP set } \\
\text { (128 probing structures) }\end{array}$} & \multicolumn{2}{|c|}{$\begin{array}{c}\text { Polarizability set }{ }^{\mathbf{a}, \mathbf{b}} \\
(70 \text { molecules })\end{array}$} \\
\hline & RMSE & 0.0563 & $\operatorname{RMSE}\left(\alpha_{i i}\right)$ & 0.68 \\
\hline & UMPE & & $\operatorname{UMPE}\left(\alpha_{i i}\right)$ & $4.2 \%$ \\
\hline & & & $\operatorname{RMSE}\left(\alpha_{i s o}\right)$ & 0.47 \\
\hline & & & $\operatorname{UMPE}\left(\alpha_{i s o}\right)$ & $3.0 \%$ \\
\hline \multirow[t]{4}{*}{ fit-esp } & RMSE & 0.0448 & $\operatorname{RMSE}\left(\alpha_{i i}\right)$ & 0.80 \\
\hline & UMPE & & $\operatorname{UMPE}\left(\alpha_{i i}\right)$ & $4.6 \%$ \\
\hline & & & $\operatorname{RMSE}\left(\alpha_{i s o}\right)$ & 0.65 \\
\hline & & & $\operatorname{UMPE}\left(\alpha_{i s o}\right)$ & $3.7 \%$ \\
\hline \multirow[t]{4}{*}{ final } & RMSE & 0.0488 & $\operatorname{RMSE}\left(\alpha_{i i}\right)$ & 0.71 \\
\hline & UMPE & & $\operatorname{UMPE}\left(\alpha_{i i}\right)$ & $4.2 \%$ \\
\hline & & & $\operatorname{RMSE}\left(\alpha_{i s o}\right)$ & 0.52 \\
\hline & & & $\operatorname{UMPE}\left(\alpha_{i s o}\right)$ & $3.1 \%$ \\
\hline
\end{tabular}
a. $\quad \alpha_{i i}$ : statistics based on three eigenvalues, where $i=x, y, z$.
b. $\alpha_{i s o}$ : statistics based on the isotropic polarizabilities, i.e., $\frac{1}{3}\left(\alpha_{x x}+\alpha_{y y}+\alpha_{z z}\right)$. 


\section{References}

1. Wilkins, D. M.; Grisafi, A.; Yang, Y.; Lao, K. U.; DiStasio, R. A.; Ceriotti, M., Accurate molecular polarizabilities with coupled cluster theory and machine learning. Proceedings of the National Academy of Sciences 2019, 116 3401-3406.

2. Řezáč, J.; Huang, Y.; Hobza, P.; Beran, G. J. O., Benchmark Calculations of Three-Body Intermolecular Interactions and the Performance of Low-Cost Electronic Structure Methods. J. Chem. Theory Comput. 2015, 11 3065-3079. 